kinases and PI3-kinase revealed that TCR independent, CD28 mediated Th2 differentiation is critically dependent on IL-4 stimulation and the activation of the MAP kinases p38 and ERK1/2. Whereas CD28 signals directly activated IL-4 and p38 in memory T cells, ERK phosphorylation required indirect stimulation by IL-2.

Conclusion The results indicate that generation of Th2 effectors requires coordinate signalling via the CD28 and IL-2 pathways. The mechanisms regulating Th2 cell differentiation might provide valuable tools for therapeutic modulation of chronic autoimmunity.

\section{THU0024 EFFECTS OF C-REACTIVE PROTEIN ON MONOCYTE ACTIVATION}

${ }^{1} \mathrm{KJ}$ Woollard, ${ }^{1} \mathrm{CJ}$ Loryman, ${ }^{2} \mathrm{GD}$ Kitas, ${ }^{1} \mathrm{HR}$ Griffiths. ${ }^{1}$ Pharmaceutical Sciences, Aston University; ${ }^{2}$ Rheumatology, Dudley Group of Hospitals, Birmingham, UK

\subsection{6/annrheumdis-2001.533}

Background FcgammaIIa (CD32) has been identified as the functional high affinity CRP receptor on leukocytes (monocytes and neutrophils). Studies on the effects of CRP on neutrophils show that receptor activation signals to downstream functional changes in phagocytosis, cytokine release and generation of reactive oxygen species. The effects of CRP on monocytes which are key players in rheumatoid chronic inflammation, have not been studied.

Objectives To investigate the phenotypic and functional consequences of CRP on peripheral blood monocytes in vitro.

Methods Peripheral whole bloods (PWB) from consenting individuals, with or without RA (ARA criteria), were collected into citrate and kept on ice. Mononuclear cells (MNC) and monocytes $(\mathrm{MON})$ were isolated by differential centrifugation using lymphoprep and Dynal negative isolation kit respectively, using sigmacoted tubes to minimise artefactual activation. Cells were exposed to CRP from 0-200 microg for up to $30 \mathrm{~min}$ at $37^{\circ} \mathrm{C}$ and analysed for (a) CD11b and CD32 expression by flow cytometry (PWB and MNC); (b) adhesion to LPS-activated human umbilical vein endothelial cells $(\mathrm{MON})$ and (c) release of reactive oxygen species (MON).

Results $\mathrm{CD} 14^{+}$ve rheumatoid monocytes expressed more than double the level of CD11b $(8.644+0.57)$, compared with those from healthy controls $(4.17+0.4), \mathrm{p}=0.0043$. There was little difference in CD32 expression. Exposure of PWB and MNC's from normal healthy subjects to increasing concentrations of CRP led to a dose dependent increase in CD11b expression from $5.7+0.1$ to $12+0.6$ at 200 microg CRP ( $p<0.01$ ); this was not enhanced by LPS $(500 \mathrm{ng} / \mathrm{ml})$. However, not all normals were responders to CRP: $46 \%$ of subjects $(n=13)$ showed no change in CD11b levels. Monocyte adhesion to HUVECs as determined using BCECF loaded cells relative to U937 monocytes, significantly altered following treatment with CRP for 30 mins at $37^{\circ} \mathrm{C}$. Release of superoxide anion (MON) was elicited by 200 but not 20 microg CRP, as determined by cytochrome C reduction.

Conclusion CRP can effect monocyte activation ex vivo, and induce phenotypic changes similar to those observed in rheumatoid patients. The variation in response from normal subjects may reflect different CD32 genotypes. A single nucleotide polymorphism in FcgammaRIIa which encodes histidine or arginine at position 131, strongly influences both IgG2a and CRP binding $^{1}$ and may identify individuals with functionally discrete responses to CRP. Allele specific response to CRP may be important in monocyte activation and extravasation during rheumatoid arthritis and complicating vascular disease.

\section{REFERENCE}

1 Stein MP. C-reactive protein binding to Fc gamma Rlla on human monocytes and neutrophils is allele specific. J Clin Invest. 2000;105:369-76

\section{Back Pain}

\section{AB0163 PLAIN RADIOGRAPHY FINDINGS IN PATIENTS WITH LOW BACK PAIN}

Y Akkoc, Y Kirazli, F Erer, G Celeboglu. Physical Medicine and Rehabilitation, Ege University, Izmir, Turkey

10.1136/annrheumdis-2001.534

\section{Background}

Objectives This study was performed to investigate the radiopathological findings based on plain radiographic examinations of patients with low back pain.

Methods In the present study, we have examined 747 patients, with a mean age of 47.97 years, $488(65.32 \%)$ were women and 259 (34.67\%) were men. Plain antero-posterior and lateral lumbosacral vertebrae radiographes were obtained from the patients and were examined in regard to the presence of congenital abnormalities (sacralisation, lumbarisation, spina bifida), lumbar degenerative disc disease, spondylolisthesis and osteoporosis. Kellgren-Lawrence's scoring system was used to assess the presence of degenerative disc disease. Increased vertebral radiolucency, accentuation of vertebral endplates and prominence of vertical trabeculae due to relative loss of horizontal trabeculae were accepted as radiological findings of osteoporosis.

Results It was found that degenerative disc disease (29.58\%) was the most frequently encountered radiopathological finding, followed by osteoporosis (23.3\%) and congenital abnormalities $(21.15 \%)$. Spondylolisthesis (6.02\%) was relatively rare. The most common congenital abnormality was sacralisation (17.67\%). Degenerative disc disease was most frequently seen at L4-L5 and L5-S1 levels. When the radiopathological findings were analysed according to sex distribution, there was no significant difference between women and men, except for osteoporosis.

Conclusion The results of this study showed that degenerative disc disease was the most frequently seen radiopathological finding, followed by osteoporosis, congenital abnormalities and spondylolisthesis in patients with low back pain.

\section{AB0164 POSTURAL SYMPTOMS AND THE EFFECT OF TREATMENT WITH PLANTARS AND/OR PRISMATIC LENSES}

A Teixeira, F Espinheira, J Landeiro, P Araújo, L Miranda, M Parente. Rheumatology, Portuguese Institut of Rheumatology, Lisbon, Portugal

10.1136/annrheumdis-2001.535

\section{Background}

Objectives To review and evaluate in a prospective study the most frequent postural symptoms and examine the effect of 